\title{
GLOBAL BOUNDS FOR THE LYAPUNOV EXPONENT AND THE INTEGRATED DENSITY OF STATES OF RANDOM SCHRÖDINGER OPERATORS IN ONE DIMENSION
}

\author{
V. KOSTRYKIN AND R. SCHRADER*
}

\begin{abstract}
In this article we prove an upper bound for the Lyapunov exponent $\gamma(E)$ and a two-sided bound for the integrated density of states $N(E)$ at an arbitrary energy $E>0$ of random Schrödinger operators in one dimension. These Schrödinger operators are given by potentials of identical shape centered at every lattice site but with non-overlapping supports and with randomly varying coupling constants. Both types of bounds only involve scattering data for the single-site potential. They show in particular that both $\gamma(E)$ and $N(E)-\sqrt{E} / \pi$ decay at infinity at least like $1 / \sqrt{E}$. As an example we consider the random Kronig-Penney model.
\end{abstract}

\section{INTRODUCTION}

In this article we will consider random Schrödinger operators $H(\omega)$ in $L^{2}(\mathbb{R})$ of the form

$$
H(\omega)=H_{0}+V_{\omega}, \quad H_{0}=-\frac{d^{2}}{d x^{2}}, \quad V_{\omega}=\sum_{j \in \mathbb{Z}} \alpha_{j}(\omega) f(\cdot-j),
$$

where $\left\{\alpha_{j}(\omega)\right\}_{j \in \mathbb{Z}}$ is a sequence of i.i.d. (independent, identically distributed) variables on a complete probability space $(\Omega, \mathcal{F}, \mathbb{P})$ having a common distribution measure $\kappa$ (i.e. $\mathbb{P}\left\{\alpha_{j} \in\right.$ $\Delta\}=\kappa(\Delta)$ for any Borel set $\Delta \subset \mathbb{R})$. In what follows we always suppose that $\kappa$ is supported on a compact interval and the single-site potential $f$ is integrable with support in the interval $[-1 / 2,1 / 2]$. Moreover, the random variables are assumed to form a stationary, metrically transitive random field, i.e. there are measure preserving ergodic transformations $\left\{T_{j}\right\}_{j \in \mathbb{Z}}$ such that $\alpha_{j}\left(T_{k} \omega\right)=\alpha_{j-k}(\omega)$ for all $\omega \in \Omega$. The spectral properties of the operator (1) were studied in detail in $[9,7,11,16,22]$. The results are most complete for the case when $f$ is the point interaction (see [1]).

The integrated density of states $N(E)$ and the Lyapunov exponent $\gamma(E)$ are important quantities associated with operators of the form (1) (see e.g. [4]). In particular, according to IshiiPastur-Kotani theorem [15] the set $\{E: \gamma(E)=0\}$ is the essential support of the absolute continuous part of the spectral measure for $H(\omega)$.

The main idea of our approach is to approximate the operator (1) by means of the sequence

$$
H^{(n)}(\omega)=H_{0}+\sum_{j=-n}^{n} \alpha_{j}(\omega) f(\cdot-j)
$$

with unchanged $H_{0}$, which converges to $H(\omega)$ in the strong resolvent sense. This differs from the usual approach where one puts the whole system in a box, which then tends to infinity (see e.g. [4]). In [12] (see also [13]) we used this approximation to invoke scattering theory for the study the spectral properties of the limiting operator (1). Some other applications of scattering

Date: May 13, 2000.

1991 Mathematics Subject Classification. (2000 Revision) Primary 82B44; Secondary 34F05, 60H25.

Key words and phrases. Random Schrödinger operators, Lyapunov exponent, density of states.

PACS Numbers. 03.65.-w, 72.15.Rn, 71.55.Jv, 71.20.-b, 73.23.-b, 72.10.Fk.

* R.S. supported in part by DFG SFB 288 "Differentialgeometrie und Quantenphysik". 
theory to the study of spectral properties of such type Schrödinger operators in one dimension can be found in [11] and [22].

One of the important ingredients of our approach developed in [12] is the Lifshitz-Krein spectral shift function. The spectral shift function naturally replaces the eigenvalue counting function usually used to construct the density of states for the operator (1). The celebrated Birman-Krein theorem (see e.g. [3]) relates the spectral shift function to scattering theory. In fact, up to a factor $-\pi^{-1}$ it may be identified with the scattering phase for the pair $\left(H^{(n)}(\omega)\right.$, $\left.H_{0}\right)$, i.e. $\xi^{(n)}(E ; \omega)=-\pi^{-1} \delta^{(n)}(E ; \omega)$ when $E>0$,

$$
\delta^{(n)}(E ; \omega)=\frac{1}{2 i} \log \operatorname{det} S^{(n)}(E ; \omega)=\frac{1}{2 i} \log \operatorname{det}\left(\begin{array}{cc}
T_{\omega}^{(n)}(E) & R_{\omega}^{(n)}(E) \\
L_{\omega}^{(n)}(E) & T_{\omega}^{(n)}(E)
\end{array}\right) .
$$

Here $\left|T^{(n)}(E)\right|^{2}$ and $\left|R^{(n)}(E)\right|^{2}=\left|L^{(n)}(E)\right|^{2}$ have the meaning of transmission and reflection coefficients, respectively, such that $\left|T^{(n)}(E)\right|^{2}+\left|R^{(n)}(E)\right|^{2}=1$. For $E<0$ the quantity $\xi^{(n)}(E ; \omega)$ equals minus the counting function for $H^{(n)}(\omega)$.

In particular in [12] we proved the almost sure existence of the limit

$$
\xi(E)=\lim _{n \rightarrow \infty} \frac{\xi^{(n)}(E ; \omega)}{2 n+1},
$$

which we called the spectral shift density. Also we proved the equality $\xi(E)=N_{0}(E)-$ $N(E)$, where $N(E)$ and $N_{0}(E)=\pi^{-1}[\max (0, E)]^{1 / 2}$ are the integrated density of states of the Hamiltonians $H(\omega)$ and $H_{0}$ respectively. This result also extends to higher dimension in the continuous [14] and discrete [5] cases. Also we showed that almost surely the Lyapunov exponent $\gamma(E)$ at energy $E>0$ is given as

$$
\gamma(E)=-\lim _{n \rightarrow \infty} \frac{\log \left|T^{(n)}(E ; \omega)\right|}{2 n+1},
$$

where $T^{(n)}(E, \omega)$ is the transmission amplitude for the pair of Hamiltonians $\left(H^{(n)}(\omega), H_{0}\right)$ at energy $E$. We recall that $\gamma(E)$ is defined as the upper Lyapunov exponent for the fundamental matrix at energy $E$ of the Schrödinger operator $H(\omega)$. The connection between the Lyapunov exponent and the transmission coefficient $\left|T_{\omega}^{(n)}(E)\right|$ was recognized long ago [17, 18]. A complete proof has appeared in [12].

We note that the theory of the spectral shift function was also recently used to show that the integrated density of states is independent of the choice of boundary conditions [19] on the sides of a large box, in which the system is put.

The conditions on the random variables $\alpha_{j}$ and the single-site potential $f$ stated above are slightly weaker than those in [12]. However the results of [12] which will be used below remain valid also in this more general case.

The aim of the present paper is to prove global bounds for the Lyapunov exponent and the integrated density of states, i.e. bounds which hold for all $E>0$ and describe the correct asymptotic behavior in the limit $E \rightarrow \infty$. These results are formulated as Theorems 1 and 2 below. To the best of our knowledge the first article to look for the asymptotic behavior of $\gamma(E)$ and $N(E)$ in the limit $E \rightarrow \infty$ is [2]. The best known estimate for the integrated density of states is due to Kirsch and Martinelli [10, Corollary 3.1]. This bound however does not reproduce the correct asymptotic behavior of $N(E)$ in the large energy limit. Another estimate, which is due to Pastur and Figotin (see [20, Sec. V.11.B]), is valid for an $\mathbb{R}$-metrically transitive random field. Since our potential $V_{\omega}(x)$ is a $\mathbb{Z}$-metrically transitive field this estimate does not apply directly to the present situation. Our two-sided estimate leads to the bound (23) below which is very close to that of Pastur and Figotin.

In what follows $C$ will denote a finite positive generic constant varying with the context, but which depends only on $f$ and $\kappa$. 
We are indebted to Leonid Pastur for reading the preliminary version of this article.

\section{THE LyAPUNOV EXPONENT}

We recall that the scattering matrix $S(E)$ for a pair of Hamiltonians $\left(H, H_{0}\right)$ on $L^{2}(\mathbb{R})$ at fixed energy $E \geq 0$ is a $2 \times 2$ unitary matrix

$$
S(E)=\left(\begin{array}{cc}
T(E) & R(E) \\
L(E) & T(E)
\end{array}\right)
$$

where $L(E)$ and $R(E)$ denote the left and right reflection amplitudes respectively. The transmission amplitude $T(E)$ can vanish only for $E=0$ (see $[8,6]$ ). To any S-matrix (4) we associate the unimodular matrix

$$
\Lambda(E)=\left(\begin{array}{cc}
\frac{1}{T(E)} & -\frac{R(E)}{T(E)} \\
\frac{L(E)}{T(E)} & \frac{1}{T(E)}
\end{array}\right) .
$$

Let $T_{\alpha}(E), R_{\alpha}(E), L_{\alpha}(E)$ be the elements of the S-matrix at energy $E$ for the pair of operators $\left(H_{0}+\alpha f, H_{0}\right)$ and $\Lambda_{\alpha}(E)$ the corresponding $\Lambda$-matrix. Also let $\widetilde{\Lambda}_{\alpha}(E)=U_{E}^{1 / 2} \Lambda_{\alpha}(E) U_{E}^{1 / 2}$ with

$$
U_{E}=\left(\begin{array}{cc}
e^{i \sqrt{E}} & 0 \\
0 & e^{-i \sqrt{E}}
\end{array}\right)
$$

Explicitly we have

$$
\widetilde{\Lambda}_{\alpha}(E)=\left(\begin{array}{cc}
\frac{e^{i \sqrt{E}}}{T_{\alpha}(E)} & -\frac{R_{\alpha}(E)}{T_{\alpha}(E)} \\
\frac{L_{\alpha}(E)}{T_{\alpha}(E)} & \frac{e^{-i \sqrt{E}}}{\overline{T(E)}}
\end{array}\right)
$$

Consider the matrix

$$
A(E)=\mathbb{E}\left\{\widetilde{\Lambda}_{\alpha(\omega)}(E)^{\dagger} \widetilde{\Lambda}_{\alpha(\omega)}(E)\right\}=\int \widetilde{\Lambda}_{\alpha}(E)^{\dagger} \widetilde{\Lambda}_{\alpha}(E) d \kappa(\alpha) \geq 0,
$$

where for brevity we write $\alpha(\omega)$ instead of $\alpha_{j}(\omega)$ with some $j \in \mathbb{Z}$. Let $\beta_{+}(E)$ be the largest eigenvalue of $A(E)$ and $\beta_{-}(E)$ the smallest. It will turn out below that $\beta_{+}(E) \geq 1$. Set $\widetilde{\gamma}(E)=\left(\log \beta_{+}(E)\right) / 2 \geq 0$.

The first main result of the present article is

Theorem 1. Given the Hamiltonian (1) and the distribution $\kappa$ for the coupling constant $\alpha$, for all $E>0$ the resulting Lyapunov exponent satisfies the upper bound

$$
\gamma(E) \leq \widetilde{\gamma}(E) .
$$

In particular $\gamma(E)$ decays at least like $1 / \sqrt{E}$ at infinity.

Proof. Let $\Lambda^{(n)}(E ; \omega)$ denote the $\Lambda$-matrix for the pair $\left(H^{(n)}(\omega), H_{0}\right)$, which by the factorization property can be represented in the form

$$
\Lambda^{(n)}(E ; \omega)=U_{E}^{-n-1 / 2} \prod_{j=-n}^{n} \widetilde{\Lambda}_{\alpha_{j}(\omega)}(E) \cdot U_{E}^{-n-1 / 2} .
$$

In fact, this factorization property is a consequence of the multiplicativity property of the fundamental matrix (see [12] for a proof and for references to earlier work). A short calculation gives

$$
\left|T^{(n)}(E ; \omega)\right|^{-2}=\frac{1}{4} \operatorname{tr}\left(\Lambda^{(n)}(E ; \omega)^{\dagger} \Lambda^{(n)}(E ; \omega)\right)+\frac{1}{2} .
$$


With $\mathbb{E}$ denoting the expectation with respect to the measure $\mathbb{P}$, by Jensen's inequality and (7) we therefore have the estimate

$$
\begin{aligned}
& e^{-2 \mathbb{E}\left\{\log \left|T^{(n)}(E ; \omega)\right|\right\}} \leq \mathbb{E}\left\{\left|T^{(n)}(E ; \omega)\right|^{-2}\right\} \\
& \quad=\frac{1}{4} \mathbb{E}\left\{\operatorname{tr}\left(\Lambda^{(n)}(E ; \omega)^{\dagger} \Lambda^{(n)}(E ; \omega)\right)\right\}+\frac{1}{2} .
\end{aligned}
$$

From the factorization property (6) it follows that

$$
\operatorname{tr}\left(\Lambda^{(n)}(E ; \omega)^{\dagger} \Lambda^{(n)}(E ; \omega)\right)=\operatorname{tr}\left(\prod_{j=n}^{-n} \widetilde{\Lambda}_{\alpha_{j}(\omega)}(E)^{\dagger} \prod_{j=-n}^{n} \widetilde{\Lambda}_{\alpha_{j}(\omega)}(E)\right) .
$$

We will now make use of the fact that the $\alpha_{k}(\omega)$ are i.i.d. random variables. For this purpose define the $2 \times 2$ matrices $A_{j}(E) \geq 0$ recursively by $A_{0}=I$ and

$$
A_{j}(E)=\int \widetilde{\Lambda}_{\alpha}(E)^{\dagger} A_{j-1}(E) \widetilde{\Lambda}_{\alpha}(E) d \kappa(\alpha),
$$

such that in particular $A(E)=A_{1}(E)$. Now it is easy to see that

$$
\begin{aligned}
\mathbb{E} & \left\{\operatorname{tr}\left(\Lambda^{(n)}(E ; \omega)^{\dagger} \Lambda^{(n)}(E ; \omega)\right)\right\} \\
& =\operatorname{tr}\left(\mathbb{E}\left(\Lambda^{(n)}(E ; \omega)^{\dagger} \Lambda^{(n)}(E ; \omega)\right)\right)=A_{2 n+1}(E) .
\end{aligned}
$$

We now use the fact that the operator inequality $0 \leq A \leq A^{\prime}$ implies $0 \leq \operatorname{tr} A \leq \operatorname{tr} A^{\prime}$ and $B^{\dagger} A B \leq B^{\dagger} A^{\prime} B$ for all $B$. In particular we have $A(E) \leq \beta_{+}(E) I$ from which we obtain the recursive estimates $A_{j}(E) \leq \beta_{+}(E) A_{j-1}(E) \leq \cdots \leq \beta_{+}(E)^{j} I$ and hence

$$
\mathbb{E}\left\{\operatorname{tr}\left(\Lambda^{(n)}(E ; \omega)^{\dagger} \Lambda^{(n)}(E ; \omega)\right)\right\} \leq 2 \beta_{+}(E)^{2 n+1} .
$$

We remark that with the same arguments one proves the lower bound

$$
2 \beta_{-}(E)^{2 n+1} \leq \mathbb{E}\left(\operatorname{tr}\left(\Lambda^{(n)}(E ; \omega)^{\dagger} \Lambda^{(n)}(E ; \omega)\right)\right) .
$$

The relation (3), the estimate (12) combined with (8) and Fatou's lemma imply now

$$
\begin{aligned}
\gamma(E) & \leq \frac{1}{2} \lim _{n \rightarrow \infty} \frac{\log \mathbb{E}\left\{\left|T^{(n)}(E ; \omega)\right|^{-2}\right\}}{2 n+1} \\
& \leq \frac{1}{2} \lim _{n \rightarrow \infty} \frac{\log \left(\beta_{+}(E)^{2 n+1} / 2+1 / 2\right)}{2 n+1}=\frac{1}{2} \log \beta_{+}(E),
\end{aligned}
$$

which proves the claim (5).

To establish the last claim of the theorem we recall the following well known estimates (see e.g. $[8,6])$

$$
\left|T_{\alpha}(E)-1\right|+\left|R_{\alpha}(E)\right| \leq C \frac{1}{\sqrt{E}}
$$

valid for all large $E>0$ uniformly for all $\alpha$ in the (compact) support of $\kappa$ for fixed $f$. Using the estimate (13) in (14) gives the estimate $\beta_{+}(E) \leq 1+C / \sqrt{E}$ for all large $E$. Since $\widetilde{\gamma}(E)=$ $\left(\log \beta_{+}(E)\right) / 2$, this concludes the proof of the theorem.

Since $\gamma(E) \geq 0$, we obviously have the inequality $\beta_{+}(E) \geq 1$ for almost all $E$. We will give now a direct independent proof of this fact and simultaneously obtain an expression for $\beta_{+}(E)$. The matrix $A(E)$ may be written in the form

$$
A(E)=\left(\begin{array}{ll}
\frac{a(E)}{b(E)} & b(E) \\
b(E)
\end{array}\right)
$$


with

$$
\begin{aligned}
& a(E)=\int\left(\frac{2}{\left|T_{\alpha}(E)\right|^{2}}-1\right) d \kappa(\alpha) \\
& b(E)=-e^{i \sqrt{E}} \int \frac{R_{\alpha}(E)}{T_{\alpha}(E)^{2}} d \kappa(\alpha) .
\end{aligned}
$$

This gives the two eigenvalues of $A(E)$ in the form

$$
\beta_{ \pm}(E)=a(E) \pm|b(E)|
$$

Obviously $a(E) \geq 1$ and hence $\beta_{+}(E) \geq 1$. In fact, $a(E)=1$ is possible if and only if $R_{\alpha}(E)=0$ for almost all $\alpha$ in the support of $\kappa$. Then also $b(E)=0$ and $\beta_{+}(E)=1$. Actually (if $\operatorname{supp} \kappa$ has at least one non-isolated point) we do not believe there are nontrivial $f$ and $E$ for which this holds but in any case for such $E$ 's the Lyapunov exponent vanishes as is easily verified (see also [12]), so this is a trivial confirmation of estimate (5) in this case. In the remaining case we trivially have $\beta_{+}(E)>1$.

As an example we consider the random Kronig - Penney model which is formally obtained from $H(\omega)$ by replacing $f$ with the Dirac $\delta$-function at the origin. Then we have (correcting for a misprint on page 232 of [12])

$$
\begin{aligned}
T_{\alpha}(E) & =\left(1+i \frac{\alpha}{2 \sqrt{E}}\right)^{-1} \\
R_{\alpha}(E) & =-i \frac{\alpha}{2 \sqrt{E}}\left(1+i \frac{\alpha}{2 \sqrt{E}}\right)^{-1}
\end{aligned}
$$

and our method still applies. This gives

$$
\begin{aligned}
& a(E)=1+\frac{\left\langle\alpha^{2}\right\rangle}{4 E} \\
& b(E)=i \frac{\langle\alpha\rangle}{2 \sqrt{E}}-\frac{\left\langle\alpha^{2}\right\rangle}{4 E} .
\end{aligned}
$$

Here for brevity by \langle\rangle we denote the mean with respect to the probability measure $\mathbb{P}$ such that

$$
\langle\alpha\rangle=\mathbb{E}\{\alpha(\omega)\}=\int \alpha d \kappa(\alpha), \quad\left\langle\alpha^{2}\right\rangle=\mathbb{E}\left\{\alpha(\omega)^{2}\right\}=\int \alpha^{2} d \kappa(\alpha) .
$$

In particular (19) gives

$$
\beta_{+}(E)=1+\frac{\left\langle\alpha^{2}\right\rangle}{4 E}+\frac{1}{2 \sqrt{E}}\left(\frac{\left\langle\alpha^{2}\right\rangle}{4 E}+\langle\alpha\rangle^{2}\right)^{1 / 2} .
$$

So also in this case $\gamma(E)$ decays at least like $1 / \sqrt{E}$ as $E \rightarrow \infty$ and at least like $1 / E$ if the mean $\langle\alpha\rangle$ of $\alpha$ vanishes, i.e. if on average the coupling constant is zero.

\section{THE INTEGRATED DENSITY OF STATES}

We denote by $\xi_{\alpha}(E)$ the spectral shift function for the pair $\left(H_{0}+\alpha f, H_{0}\right)$. The second main result of this article is given by

Theorem 2. For all $E>0$ the spectral shift density $\xi(E)$ for the operator (1) satisfies the following two-side bound

$$
\mathbb{E}\left\{\xi_{\alpha(\omega)}(E)\right\}-r(E) \leq \xi(E) \leq \mathbb{E}\left\{\xi_{\alpha(\omega)}(E)\right\}+r(E),
$$

where

$$
r(E)=\min \left\{\frac{1}{2}, \frac{1}{\pi} \mathbb{E}\left\{\frac{\mid R_{\alpha(\omega)}(E)}{1-\left|R_{\alpha(\omega)}(E)\right|}\right\}\right\} .
$$

In particular $\mathbb{E}\left\{\xi_{\alpha(\omega)}(E)\right\}$ and $r(E)$ decays at least like $1 / \sqrt{E}$ at infinity. 
Remarks. 1. One can easily prove the following estimate

$$
\mathbb{E}\left\{\xi_{\alpha(\omega)}(E)\right\}-1 \leq \xi(E) \leq \mathbb{E}\left\{\xi_{\alpha(\omega)}(E)\right\}+1,
$$

which is valid for all $E \in \mathbb{R}$.

2. By the monotonicity of the spectral shift function with respect to perturbation $\xi(E) \geq 0$ if supp $\kappa \subset \mathbb{R}_{+}$and $\xi(E) \leq 0$ if supp $\kappa \subset \mathbb{R}_{-}$for almost all $E>0$.

3. For large $E>0$ by (13)

$$
r(E)=\min \left\{\frac{1}{2}, \frac{1}{\pi} \mathbb{E}\left\{\frac{\mid R_{\alpha(\omega)}(E)}{1-\left|R_{\alpha(\omega)}(E)\right|}\right\}\right\}=\frac{1}{\pi} \mathbb{E}\left\{\frac{\mid R_{\alpha(\omega)}(E)}{1-\left|R_{\alpha(\omega)}(E)\right|}\right\} \leq \frac{C}{\sqrt{E}} .
$$

4. In [12] we proved the relation $\xi(E)=N_{0}(E)-N(E)=\sqrt{E} / \pi-N(E)$, where $N_{0}(E)$ is the integrated density of states for the free operator $H_{0}$. Theorem 2 then gives the following two-sided bound for the integrated density of states

$$
\frac{\sqrt{E}}{\pi}-\mathbb{E}\left\{\xi_{\alpha(\omega)}(E)\right\}-r(E) \leq N(E) \leq \frac{\sqrt{E}}{\pi}-\mathbb{E}\left\{\xi_{\alpha(\omega)}(E)\right\}+r(E), \quad E>0 .
$$

There are some other upper bounds on the integrated density of states. A well-known result is a one-sided bound due to Kirsch and Martinelli [10, Corollary 3.1],

$$
N(E) \leq \frac{C}{\sqrt{\eta}} \mathbb{E}\left\{\int_{-1 / 2}^{1 / 2}\left(E+\eta-V_{\omega}(x)\right)_{+} d x\right\}
$$

for any $\eta>0$ and all $E \in \mathbb{R}$. This bound however does not reproduce the correct asymptotic behavior of $N(E)$ in the large energy limit.

5. The bounds (5) and (22) are of interest in the context of the Thouless formula (see e.g. [20])

$$
\gamma(E)-\gamma_{0}(E)=-\int_{\mathbb{R}} \log \left|E-E^{\prime}\right| d \xi\left(E^{\prime}\right), \quad E \in \mathbb{R}
$$

where $\gamma_{0}(E)=[\max (0,-E)]^{1 / 2}$ is the Lyapunov exponent for $H_{0}$. The Thouless formula in the form (24) can be viewed as a subtracted dispersion relation (see e.g. [12]).

Proof. In [12] we proved (see Theorem 3.3 there and its proof) that for any two potentials $V_{1}$ and $V_{2}$ with (compact) disjoint supports one has

$$
\xi\left(E ; H_{0}+V_{1}+V_{2}, H_{0}\right)=\xi\left(E ; H_{0}+V_{1}, H_{0}\right)+\xi\left(E ; H_{0}+V_{2}, H_{0}\right)+\xi_{12}(E)
$$

with

$$
\xi_{12}(E)=-\frac{1}{2 \pi i} \log \frac{1-R_{1}(E)}{1-\overline{R_{1}(E)}} \frac{L_{2}(E)}{L_{2}(E)},
$$

where $R_{k}(E)$ and $L_{k}(E)$ are the right and left reflection coefficients for the Schrödinger equation with the potential $V_{k}, k=1,2$. Actually Theorem 3.3 in [12] states that $\left|\xi_{12}(E)\right| \leq 1 / 2$ for all $E \geq 0$. Now we improve on this estimate. As in [12] we set

$$
L_{k}(E)=a_{k}(E) e^{i \delta_{k}^{(L)}}, \quad R_{k}(E)=a_{k}(E) e^{i \delta_{k}^{(R)}}, \quad k=1,2
$$


with $0 \leq a_{k}(E) \leq 1$. Moreover $a_{k}(E)=1$ only when $T_{k}(E)=0$, which we recall can happen only if $E=0$. Therefore

$$
\begin{aligned}
& \log \frac{1-R_{1}(E) L_{2}(E)}{1-\overline{R_{1}(E)} \overline{L_{2}(E)}} \\
& =\log \frac{1-a_{1}(E) a_{2}(E) e^{i\left(\delta_{1}^{(R)}+\delta_{2}^{(L)}\right)}}{1-a_{1}(E) a_{2}(E) e^{-i\left(\delta_{1}^{(R)}+\delta_{2}^{(L)}\right)}} \\
& =-2 i \arctan \frac{a_{1}(E) a_{2}(E) \sin \left(\delta_{1}^{(R)}+\delta_{2}^{(L)}\right)}{1-a_{1}(E) a_{2}(E) \cos \left(\delta_{1}^{(R)}+\delta_{2}^{(L)}\right)} .
\end{aligned}
$$

By means of the inequality $|\arctan x| \leq|x|$ we immediately obtain

$$
\left|\xi_{12}(E)\right| \leq \min \left\{\frac{1}{2}, \frac{1}{\pi} \frac{a_{1}(E) a_{2}(E)}{1-a_{1}(E) a_{2}(E)}\right\} .
$$

Since $0 \leq a_{k}(E)<1$ we can replace $a_{1}(E) a_{2}(E)\left(1-a_{1}(E) a_{2}(E)\right)^{-1}$ either by $a_{1}(E)(1-$ $\left.a_{1}(E)\right)^{-1}$ or by $a_{2}(E)\left(1-a_{2}(E)\right)^{-1}$.

Now let us consider the operator $H^{(n)}(\omega)$ for finite $n$. Applying the inequality (25) we obtain

$$
\begin{aligned}
& \left|\xi^{(n)}(E ; \omega)-\xi_{\alpha_{n}(\omega)}(E)-\xi_{\alpha_{-n}(\omega)}(E)-\xi^{(n-1)}(E ; \omega)\right| \\
& \quad \leq \min \left\{\frac{1}{2}, \frac{1}{\pi} \frac{\left|R_{\alpha_{n}(\omega)}(E)\right|}{1-\left|R_{\alpha_{n}(\omega)}(E)\right|}\right\}+\min \left\{\frac{1}{2}, \frac{1}{\pi} \frac{\left|R_{\alpha_{-n}(\omega)}(E)\right|}{1-\left|R_{\alpha_{-n}(\omega)}(E)\right|}\right\}
\end{aligned}
$$

Repeating this procedure recursively we obtain

$$
\left|\xi^{(n)}(E ; \omega)-\sum_{j=-n}^{n} \xi_{\alpha_{j}(\omega)}(E)\right| \leq \sum_{j=-n}^{n} \min \left\{\frac{1}{2}, \frac{1}{\pi} \frac{\left|R_{\alpha_{j}(\omega)}(E)\right|}{1-\left|R_{\alpha_{j}(\omega)}(E)\right|}\right\} .
$$

From the existence of the spectral shift density (2) by the Birkhoff ergodic theorem it follows that

$$
\left|\xi(E)-\mathbb{E}\left\{\xi_{\alpha(\omega)}(E)\right\}\right| \leq \mathbb{E}\left\{\min \left\{\frac{1}{2}, \frac{1}{\pi} \frac{\left|R_{\alpha(\omega)}(E)\right|}{1-\left|R_{\alpha(\omega)}(E)\right|}\right\}\right\} .
$$

From the obvious inequality

$$
\mathbb{E}\left\{\min \left\{\frac{1}{2}, \frac{1}{\pi} \frac{\left|R_{\alpha(\omega)}(E)\right|}{1-\left|R_{\alpha(\omega)}(E)\right|}\right\}\right\} \leq \min \left\{\frac{1}{2}, \frac{1}{\pi} \mathbb{E}\left\{\frac{\left|R_{\alpha(\omega)}(E)\right|}{1-\left|R_{\alpha(\omega)}(E)\right|}\right\}\right\}
$$

the bound (22) follows.

For large $E$ we have the following asymptotics [6] uniformly in $\alpha$ on compact sets:

$$
\begin{aligned}
R_{\alpha}(E) & =\frac{\alpha}{2 i \sqrt{E}} \int_{\mathbb{R}} e^{2 i \sqrt{E} t} f(t) d t+O\left(E^{-1}\right), \\
L_{\alpha}(E) & =\frac{\alpha}{2 i \sqrt{E}} \int_{\mathbb{R}} e^{-2 i \sqrt{E} t} f(t) d t+O\left(E^{-1}\right)
\end{aligned}
$$

such that $R_{\alpha}(E)=O(1 / \sqrt{E})$ and $L_{\alpha}(E)=O(1 / \sqrt{E})$. If the single-site potential $f$ has $p$ derivatives in $L^{1}(\mathbb{R})$ then $L_{\alpha}(E)=O\left(E^{-(p+1) / 2}\right)$ and $R_{\alpha}(E)=O\left(E^{-(p+1) / 2}\right)$ as $E \rightarrow \infty$ [6]. The estimate $\mathbb{E}\left\{\xi_{\alpha(\omega)}(E)\right\}=O(1 / \sqrt{E})$ is Proposition 3 below.

As an example we consider again the random Kronig-Penney model. The single-site spectral shift function is given in this case by

$$
\xi_{\alpha}(E)=\frac{1}{\pi} \arctan \left(\frac{\alpha}{2 \sqrt{E}}\right), \quad E>0 .
$$


Therefore

and thus

$$
\mathbb{E}\left\{\xi_{\alpha(\omega)}(E)\right\}=\frac{1}{\pi} \int_{\mathbb{R}} \arctan \left(\frac{\alpha}{2 \sqrt{E}}\right) d \kappa(\alpha)
$$

$$
\left|\mathbb{E}\left\{\xi_{\alpha(\omega)}(E)\right\}\right| \leq \frac{\langle|\alpha|\rangle}{2 \pi \sqrt{E}}
$$

Using the explicit expression for the reflection amplitude one can easily show that

$$
\frac{\langle|\alpha|\rangle}{2 \sqrt{E}}+\frac{\left\langle\alpha^{2}\right\rangle}{4 E} \leq \mathbb{E}\left\{\frac{\left|R_{\alpha(\omega)}(E)\right|}{1-\left|R_{\alpha(\omega)}(E)\right|}\right\} \leq \frac{\langle|\alpha|\rangle}{2 \sqrt{E}}+\frac{\left\langle\alpha^{2}\right\rangle}{2 E} .
$$

We complete this section with an estimate on $\mathbb{E}\left\{\xi_{\alpha(\omega)}(E)\right\}$ in the general case. We will prove

Proposition 3. There is a constant $c>0$ independent of $E, f$, and $\kappa$ such that for all $E>0$

$$
\left|\mathbb{E}\left\{\xi_{\alpha(\omega)}(E)\right\}\right| \leq \frac{C}{2 \sqrt{E}} \mathbb{E}\left\{|\alpha(\omega)|^{1 / 2}\right\}^{2} \int_{-1 / 2}^{1 / 2}|f(x)| d x .
$$

Let $l^{1 / 2}\left(L^{1}\right)$ denote the Birman-Solomyak class of measurable functions $V$ for which

$$
\|V\|_{l^{1 / 2}\left(L^{1}\right)}=\left[\sum_{j=-\infty}^{\infty}\left(\int_{j-1 / 2}^{j+1 / 2}|V(x)| d x\right)^{1 / 2}\right]^{2}<\infty .
$$

The claim of the proposition immediately follows from the following

Lemma 4. Let $V \in l^{1 / 2}\left(L^{1}\right)$. There is a constant $c_{1}$ independent of $V$ and $E$ such that

$$
\left|\xi\left(E ; H_{0}+V, H_{0}\right)\right| \leq \frac{c_{1}}{2 \sqrt{E}}\|V\|_{l^{1 / 2}\left(L^{1}\right)}
$$

for all $E>0$.

Proof. As proved in [23] there is a constant $c_{2}>0$ independent of $E$ and $V$ such that

$$
\left|\xi\left(E ; H_{0}+V, H_{0}\right)\right| \leq C_{1}\left\|V^{1 / 2} R_{0}(E+i 0)|V|^{1 / 2}\right\|_{\mathcal{J}_{1}},
$$

where $V^{1 / 2}=\operatorname{sign} V|V|^{1 / 2}, R_{0}(z)=\left(H_{0}-z\right)^{-1}$, and $\|\cdot\|_{\mathcal{J}_{1}}$ denotes the trace class norm (see e.g. [21]). From the proof of Proposition 5.6 in [21] it follows that

$$
\left\|V^{1 / 2} R_{0}(E+i 0)|V|^{1 / 2}\right\|_{\mathcal{J}_{1}} \leq \frac{c_{3}}{\sqrt{E}}\|V\|_{l^{1 / 2}\left(L^{1}\right)}
$$

for all $E>0$.

\section{REFERENCES}

[1] S. Albeverio, F. Gesztesy, R. Høegh-Krohn and H. Holden, Solvable Models in Quantum Mechanics, Springer, Berlin, 1988.

[2] L. Arnold, G. Papanicolaou and V. Wihstutz, Asymptotic analysis of the Lyapunov exponent and rotation number of the random oscillator and applications, SIAM J. Appl. Math. 46, 427 - 450 (1986).

[3] M.Sh. Birman and D.R. Yafaev, The spectral shift function. The work of M.G.Krein and its further development, St. Petersburg Math. J. 4, 833 - 870 (1993).

[4] R. Carmona and J. Lacroix, Spectral Theory of Random Schrödinger Operators, Boston, Birkhäuser, 1990.

[5] A. Chahrour, Densité intégrée d'états surfaciques et fonction généralisée de déplacement spectral pour un operateur de Schroedinger surfacique ergodique, Helv. Phys. Acta 72, 93 - 122 (1999).

[6] P. Deift and E. Trubowitz, Inverse scattering on the line, Commun. Pure Appl. Math. 32, 121 - 251 (1979).

[7] F. Delyon, B. Simon, and B. Souillard, From power pure point to continuous spectrum in disordered systems, Ann. Inst. Henri Poincaré, Phys. theor. 42, 283 - 309 (1985).

[8] L.D. Faddeev, Properties of the S-matrix of the one dimensional Schrödinger equation, AMS Transl. Ser. (2) 65, 139 - 166 (1967)

[9] W. Kirsch and F. Martinelli, On the spectrum of Schrödinger operators with a random potential, Commun. Math. Phys. 85, 329 - 350 (1982).

[10] W. Kirsch and F. Martinelli, On the density of states of Schrödinger operators with a random potential, J. Phys. A: Math. Gen. 15, 2139 - 2156 (1982). 
[11] W. Kirsch, S. Kotani, and B. Simon, Absence of absolutely continuous spectrum for some one dimensional random but deterministic operators, Ann. Inst. Henri Poincaré, Phys. theor. 42, 383 - 406 (1985).

[12] V. Kostrykin and R. Schrader, Scattering theory approach to random Schrödinger operators in one dimension, Rev. Math. Phys. 11, 187 - 242 (1999).

[13] V. Kostrykin and R. Schrader, One-dimensional disordered systems and scattering theory, preprint (1998); available from http: //www-sfb288. math.tu-berlin. de/abstractNew/337.

[14] V. Kostrykin and R. Schrader, The density of states and the spectral shift density of random Schrödinger operators, Rev. Math. Phys. (to appear); available from http: / / www . ma . utexas . edu/mp_arc/ as 99-239.

[15] S. Kotani, Ljapunov indices determine absolutely continuous spectra of stationary random one-dimensional Schrödinger operators, in K. Ito (Ed.) Stochastic Analysis, North-Holland Mathematical Library, Vol. 32, Amsterdam, North Holland, 1984, pp. 225 - 247.

[16] S. Kotani and B. Simon, Localization in general one-dimensional random systems, Commun. Math. Phys. 112, 103 - 119 (1987).

[17] I.M. Lifshitz, S.A. Gredeskul, and L.A. Pastur, Theory of the passage of particles and waves through randomly inhomogeneous media, Sov. Phys. JETP 56, 1370 - 1378 (1982).

[18] I.M. Lifshitz, S.A. Gredeskul, and L.A. Pastur, Introduction to the Theory of Disordered Systems, Wiley, New York, 1988.

[19] S. Nakamura, A remark on the Dirichlet-Neuman decoupling and the integrated density of states, preprint (2000); available from http: / / www . ma.utexas .edu/mp_arc/ as 00-121.

[20] L. Pastur and A. Figotin, Spectra of Random and Almost-Periodic Operators, Springer, Berlin, 1992.

[21] B. Simon, Trace Ideals and Their Applications, Cambridge University Press, New York, 1979.

[22] R. Sims and G. Stolz, Localization in one dimensional random media: a scattering theoretic approach, Preprint (2000); available from http: / / www . ma . utexas . edu/mp_arc/ as 00-64.

[23] A.V. Sobolev, Efficient bounds for the spectral shift function, Ann. Inst. Henri Poincaré, Phys. theor. 58, 55 83 (1993).

[24] G. Stolz, Non-monotoneous random Schrödinger operators: the Anderson model, Preprint (1999); available from http: / / www. ma.utexas.edu/mp_arc/ as 99-259.

VAdim Kostrykin, Fraunhofer-Institut Für Lasertechnik, Steinbachstrasse 15, D-52074, AACHEN, GERMANY

E-mail address: kostrykin@t-online.de, kostrykin@ilt.fhg.de

Robert Schrader, Institut FÜr Theoretische Physik, Freie Universität Berlin, ArnimalleE 14, D-14195 BERLIN, GERMANY

E-mail address: schrader@physik. fu-berlin.de 